\title{
HOU Reconstruction \& Simulation (HOURS): a simulation and reconstruction package for neutrino telescopy
}

\author{
A. G. Tsirigotis*, G. Bourlis, A. Leisos, D. Lenis and S.E. Tzamarias \\ School of Science \& Technology, Hellenic Open University \\ Tsamadou 13-15, Patra, Greece \\ E-mail: tsirigotis@eap.gr, bourlis@eap.gn, leisosceap.gr \\ tzamariadeap.gr, dlenis@eap.gr
}

The Hellenic Open University Reconstruction \& Simulation (HOURS) software package has been developed in order to study in detail the response of underwater neutrino telescopes. HOURS comprises a realistic simulation package of the detector response, including an accurate description of all the relevant physical processes, the production of signal and background as well as several analysis strategies for triggering and pattern recognition, event reconstruction, tracking and energy estimation. Furthermore, this package provides the tools for simulating calibration techniques as well as other studies to estimate the detector sensitivity to several astrophysical neutrino sources and atmospheric neutrino oscillation parameters.

XV International Workshop on Neutrino Telescopes

March 11-15 2013

Venice, Italy

\footnotetext{
* Speaker.
} 


\section{Introduction}

HOURS is one of the main physics analysis packages used extensively in evaluating architectures and technologies proposed during the design study and preparatory phase of KM3NeT [1]. Neutrino telescope performance results produced using HOURS are in good agreement with the results produced by other simulation efforts within the KM3NeT collaboration. We report on the structure and performance of the simulation package. We also report on results, using HOURS, concerning the evaluation of the performance of a Mediterranean neutrino telescope to discover/observe astrophysical neutrino sources, and on results concerning the detection efficiency of low energy atmospheric neutrinos for neutrino oscillation studies.

\section{Monte Carlo Simulation}

The HOURS physics analysis package for $\mathrm{km}^{3}$ neutrino telescopy contains physics event generators, neutrino interaction simulation, Cherenkov emission from the produced charged particles, detector response simulation, filter-triggering algorithms and reconstruction analysis code.

\subsection{Event generation}

The first step in producing the physics signal of an underwater neutrino telescope is the generation of secondaries of the neutrino interactions in the detector medium, as well as the generation of atmospheric muons that reach the detector. In HOURS the atmospheric muon background is described either by Extensive Air Showers simulations using the CORSIKA software package [2], or from atmospheric muon bundle parameterizations using the MUPAGE software package [3]. In the latter case the muons are created at the surface of a can surrounding the detector, while in the former case the muons are registered by CORSIKA at the sea surface, and afterwards are transported to the detector neighborhood taking into account energy losses.

For the simulation of neutrino interactions the primary momentum vector is generated according to phenomenological models that describe the flux from several type of astrophysical sources or from atmospheric neutrinos. The probability of upgoing neutrinos to interact with earth before reaching the detector neighborhood is not negligible for neutrino energies above $10 \mathrm{TeV}$. This Earth shadowing is taken into account in the generation of the neutrinos arriving at the detector neighborhood. For the Earth matter density as a function of planetary radius the Preliminary Reference Earth Model is used [4].

In HOURS all flavor neutrino interactions with matter are described using the appropriate cross-sections, while the generation of secondary particles is described either by PYTHIA for neutrino energies above $100 \mathrm{GeV}$ [5], or by the GENIE neutrino interaction generator for lower energies [6].

\subsection{Detector description}

In HOURS the underwater neutrino detector geometry, as well as the sea water and Earth crust compositions are described using the Geometry Description Markup Language [7]. Particle transportation and all the relevant interactions of the secondary particles produced by the neutrino interaction are described using the tools offered by the GEANT4 simulation package [8]. During 
the simulation of the detector signal produced by the secondary particles, the sea volume around the neutrino telescope is divided in two regions. The first region is a can enclosing the detector instrumented volume and extending up to $300 \mathrm{~m}$ away from the outermost optical modules, while the second region is the sea water volume beyond this can. In the can volume all particles are tracked by the GEANT4 tracking routines producing more secondary particles and Cherenkov optical photons, while in the second region only long range particles that are able to reach the can are tracked. Long range particles are muons and tau leptons.

\subsection{Optical photon generation and transportation}

Every charged particle with sufficient energy produces Cherenkov photons. These optical photons travel through the sea water and can be absorbed or scattered. The optical photon absorption and scattering lengths as a function of the photon wavelength has been measured for the various installation sites of the future Mediterranean neutrino telescope and is used in the HOURS software package. The scattering angle distribution is modeled as a combination of Rayleigh (molecular) and Mie (particulate) scattering.

Events containing high energy particles produce very large amount of Cherenkov photons. As a result the full tracking of every optical photon is very CPU time consuming for high energy neutrino interaction events or events containing high energy atmospheric muons. For this reason a fast simulation process is used in the HOURS software package. In the context of this fast simulation process, the GEANT4 code responsible for the emission of optical photons due to the Cherenkov effect is not used for high energy events. Instead, for such events an algorithm that describes the flux of optical photons and their arrival time distribution on an optical module has been developed. This flux is parameterized for various relative positions of the optical module with respect the position and direction of the track producing the Cherenkov photons. Such parameterizations are used for different kind of Cherenkov photon emitters, like electromagnetic showers initiated by $e^{-}, e^{+}, \gamma$, hadronic showers initiated by long lived hadrons and muon tracks. The parameterizations are implemented using multidimensional tables.

\subsection{Optical background and Optical Module simulation}

The photo-sensor unit of KM3NeT is a digital optical module (DOM) consisting of a 17 inch diameter pressure resistant glass sphere housing 313 -inch photomultiplier tubes. The segmentation of the photocathode area in such a Multi-PMT Optical Module will aid in distinguishing singlephoton from multi-photon hits, and thus provide a better optical background rejection and trigger efficiency. The main optical background source, which contaminates significantly the experimental signal detected by the DOM, comes from the ${ }^{40} K$ decay. The decay products, an $e^{-}$or $\gamma$, produce Cherenkov photons and if close enough more than one 3-inch PMT can be activated by a single decay. This correlated background noise, as well as uncorrelated hits from distinct decays are included in the simulation. The simulation of the PMT response to optical photons takes into account all functional characteristics of the PMT, as is described in Ref. [9].

\section{Event Reconstruction}

The reconstruction of the produced muon from the interaction of a high energy $v_{\mu}$ is described 


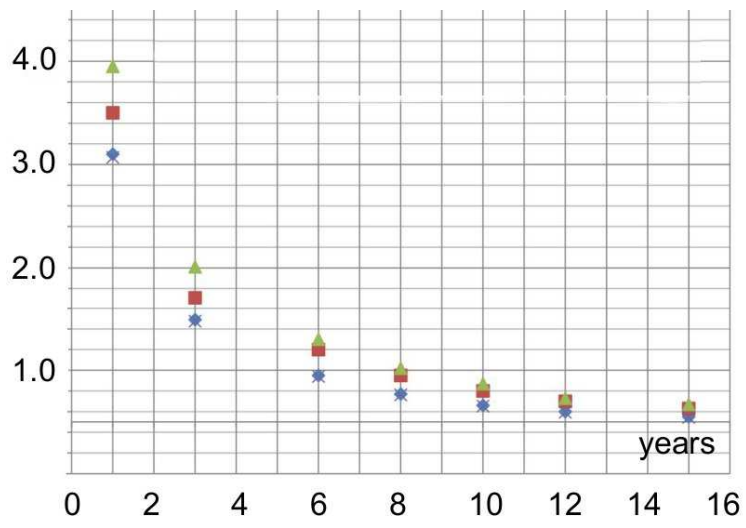

Figure 1: Discovery potential ( $5 \sigma$ with $50 \%$ probability), in units of the reference flux, as a function of the number of observation years of RXJ1713.7-3946. Points correspond to various analysis techniques. The best technique give about 5 years for discovery of the reference flux.

in detail in Ref. [9]. The reconstruction algorithm estimates the direction and energy of the muons that transverse the detector volume, and has been extended to low energy muons produced from atmospheric neutrinos interacting inside the instrumented detector volume. The number of emitted Cherenkov photons per unit length of a muon track grows rapidly with energy for high energy muons $(>1 \mathrm{TeV})$ due to radiative processes. On the contrary, muon losses due to ionization, which is the dominant process for low energy muons, grow very slowly with energy. As a result, the number of detected photons per unit muon track length cannot be used for the energy estimation of low energy muons. In this case the energy estimation technique used in HOURS is based on the estimation of the muon track length.

\section{Studies to estimate the detector sensitivity to astrophysical neutrino sources}

Observations by gamma ray telescopes have revealed many astrophysical objects, in which high-energy processes at and beyond the TeV level take place. One such source, that has been extensively studied, is the Supernova remnant RXJ1713.7-3946. It is visible $75 \%$ of the time by a Mediterranean neutrino telescope and has been found to be active in low (Fermi-LAT) and high (HESS) energy gamma rays. Assuming that the high energy gamma rays are of hadronic origin, the neutrino flux (reference flux) can be predicted by phenomenological models [10]. The potential of a Mediterranean neutrino telescope, consisting of 12320 DOMs instrumenting $3.8 \mathrm{~km}^{3}$ volume, to discover the reference flux is approximately 5 years as is presented in Fig. 1 .

\section{Studies to estimate the detector sensitivity to low energy atmospheric neutrinos}

With the measurement of a non zero value of the $\theta_{13}$ neutrino mixing parameter, interest in neutrinos as source of the baryon asymmetry of the universe has increased. Among the measurements of a rich and varied program in near future neutrino physics is the determination of the mass hierarchy. Studies into the feasibility to determine the mass hierarchy with a densely instrumented undersea neutrino detector using technology developed for KM3NeT, are ongoing. These studies 


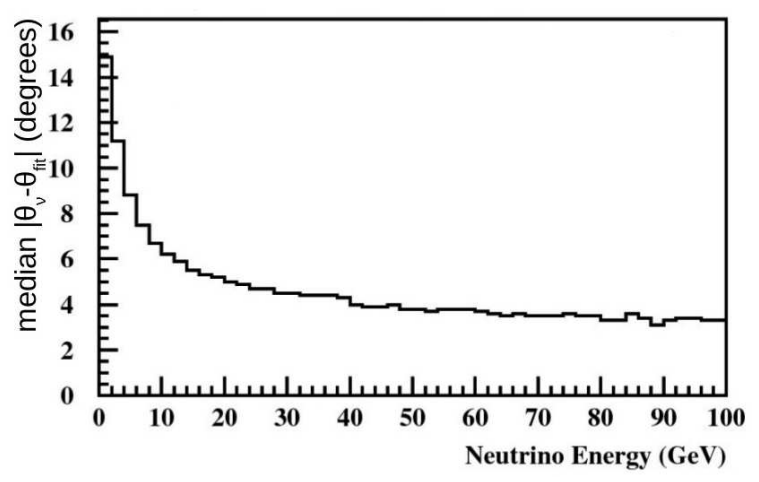

Figure 2: Median error on the reconstructed zenith angle as a function of the neutrino energy.

utilize the MSW effect on atmospheric neutrino oscillations. For the determination of the mass hierarchy the detector must have optimal performance in the required 5-10 GeV neutrino energy regime. The studies include new tracking and interaction identification algorithms for such low energy neutrinos. As described in Section 3 , the reconstruction algorithms in HOURS have been extended for the reconstruction of low energy neutrino events. Fig. 2 presents the zenith angular resolution for upgoing atmospheric $v_{\mu}$ events reconstructed from the signal detected by a small volume neutrino telescope. This telescope consists of 1000 DOMs instrumenting 1.75 Mtons of sea water.

\section{Acknowledgments}

This research has been co-financed by the European Union (European Social Fund-ESF) and Greek national funds through the Operational Program "Education and Lifelong Learning" of the National Strategic Reference Framework (NSRF)-Research Funding Program:

THALES. Investing in knowledge society through the European Social Fund.

\section{References}

[1] P. Bagley, et al. [KM3NeT consortium], Technical Design Report for a Deep-Sea Research Infrastructure in the Mediterranean Sea Incorporating a Very Large Volume Neutrino Telescope (2010), ISBN 978-90-6488-033-9

[2] J. Wentz, et al., Phys. Rev. D 67 (2003) 073020 [arXiv:hep-ph/0301199].

[3] G. Carminati, et al., Computer Physics Communications, 179 (2008) 915.

[4] A.M. Dziewonski, et al., Phys. Earth Plan. Int. 25 (1981) 297.

[5] T. Sjostrand, et al., JHEP 0605 (2006) 026 [arXiv:hep-ph/0603175].

[6] C. Andreopoulos et al., Nucl. Instr. and Meth. A 614 (2010) 87.

[7] R. Chytracek, et al., IEEE Trans. Nucl. Sci., 53 (2006) 2892.

[8] S. Agostinelli, et al., Nucl. Instr. and Meth. A 506 (2003) 250.

[9] A.G. Tsirigotis, et al., Nucl. Instr. and Meth. A 626 (2011) S185.

[10] S.R. Kelner, et al., Phys. Rev D 74 (2006) 034018. 